See discussions, stats, and author profiles for this publication at: https://www.researchgate.net/publication/324022055

\title{
Beveled needle position and pose estimation based on optical coherence tomography in ophthalmic microsurgery
}

Conference Paper · December 2017

DOI: 10.1109/ROBIO.2017.8324435

CITATIONS

0

8 authors, including:

(2)

Mingchuan Zhou

Technische Universität München

17 PUBLICATIONS 66 CITATIONS

SEE PROFILE

Hessam Roodaki

Carl Zeiss Meditec

16 PUBLICATIONS 51 CITATIONS

SEE PROFILE

Some of the authors of this publication are also working on these related projects:

Cataract surgery and prevention View project

Co-authoring book chapters of the textbook on "Pediatric Lens Diseases" View project
READS

63

Kai Huang

University of Science and Technology Beijing

141 PUBLICATIONS 988 CITATIONS

SEE PROFILE

,

Haotian Lin

Zhongshan Ophthalmic CenterSun Yat-Sen University

234 PUBLICATIONS 1,471 CITATIONS

SEE PROFILE 


\title{
Beveled Needle Position and Pose Estimation based on Optical Coherence Tomography in Ophthalmic Microsurgery
}

\author{
Mingchuan Zhou ${ }^{1}$, Kai Huang ${ }^{2}$, Abouzar Eslami ${ }^{3}$, Hessam Roodaki ${ }^{1}$, Haotian Lin $^{4}$, \\ Chris P. Lohmann ${ }^{5}$, Alois Knoll ${ }^{1}$ and M. Ali Nasseri ${ }^{5}$ \\ ${ }^{1}$ Department of Informatics, Technical University of Munich \\ Email: zhoum,knoll@in.tum.de, he.roodaki@tum.de \\ ${ }^{2}$ School of Data and Computer Science, Sun Yat-Sen University \\ Email: huangk36@mail.sysu.edu.cn \\ ${ }^{3}$ Medical Technology Business Group, Carl Zeiss Meditec AG \\ Email: abouzar.eslami@zeiss.com \\ ${ }^{4}$ Zhongshan Ophthalmic Center, Sun Yat-Sen University \\ Email: linht5@mail.sysu.edu.cn \\ ${ }^{5}$ Klinik und Poliklinik für Augenheilkunde am Klinikum rechts der Isar der, Technical University of Munich \\ Email: chris.lohmann,nasseri@mri.tum.de,
}

\begin{abstract}
The needle position and pose is essential information in ophthalmic microsurgery. Estimation of these parameters is still an open problem, which hinders the application of various robot-assisted surgeries, such as autonomous injection. This paper presents a method to estimate the position and pose of beveled needle using three-dimensional Optical Coherence Tomography (OCT) image cube. The incomplete 3D point cloud of beveled needle obtained from OCT image cube is used to reconstruct the needle by fitting the projection of needle points based on its geometric features. We demonstrate the applicability of our method in ophthalmic injection surgery using ex vivo pig eyes and evaluate the accuracy of position and pose information using a micromanipulator.
\end{abstract}

\section{INTRODUCTION}

The present situation of eye pathologies, which contributes to more than 280 million visual impairments [1], raises an increasing demand for the ophthalmic surgery. Vitreoretinal surgery is a typical ophthalmic surgery consisting of complex manual tasks. The incisions, created by keratome and trocar at the sclera in a circle and $3.5 \mathrm{~mm}$ away from the cornea center, are made to provide the entrance for three tools: light source, surgical tool, and irrigation cannula [2]. The irrigation cannula is used for liquid injection to maintain appropriate intraocular pressure (IOP). The light source is used to illuminate the intended area on the retina, allowing the planar view of the area obtained and analyzed by surgeons through the microscope. The surgical progress proposes a great challenge of delicate operation and sensitive perception to surgeons.

Among various ophthalmic surgery routines, the beveled needle is used to deliver drug to the particular part of eye. A high potential treatment for a disease called Retinal Vein Occlusion (RVO) is to inject clot-dissolving drugs directly into the affected vessel [3]. In robot assisted ophthalmic surgery, the accurate position and pose information of needle is essential for guided navigation and injection, which is hard to estimate via kinematics due to the very thin, long and relatively flexible needle body [4].
Optical Coherence Tomography (OCT), which is originally used for ophthalmic diseases diagnosis for its suitable resolution, has been developed to give real-time information about interactions between the surgical tool and intraocular tissue. Microscope-mounted intraoperative OCT solution developed by Carl Zeiss Meditec (RESCAN700), firstly described in clinical use in 2014 [5], can share the same optical path with the surgical microscope and give real-time cross section information, which is an ideal imaging modality for ophthalmic surgery.

This paper introduces OCT to estimate the position and pose information for the beveled needle in ophthalmic surgery for the first time. Here, we face following challenges. Firstly, the iterative closest point (ICP) algorithm [6] is not suitable for our goal. Our goal is more inclined to estimate the dimension of needle, because medical image processing has a high safety critical requirement and the inherent size of object can be adopted as a judgment for the estimation result. Nevertheless, ICP needs CAD model in advance to sample the model point cloud and then match to object point cloud [7]. Secondly, due to the imaging principle of OCT, only the upper surface points of needle are obtained, and there are also deformations existing in some section images because of reflection. To solve these problems, the projection from the needle bounding boxes for each section image is used to fit with one ellipse and two lines. Based on the geometric properties of the needle, these planar fitting results can be mapped back to 3D space which can fully reconstruct the needle. The contributions of this work are summarized as follows.

- A framework for estimating needle position and pose is proposed using OCT for ophthalmic microsurgery based on the geometric feature of beveled needle.

- The experimental evaluations on ex vivo pig eyes demonstrate that the method has a good performance of testretest reliability and position estimation accuracy can be controlled within $10 \mu \mathrm{m}$.

The rest of this paper is organized as follows: Section II 
briefly presents related work. The proposed method is introduced in Section III. Then Section IV gives the experimental evaluations to prove the effectiveness of our proposed method. Section V concludes this paper with the discussion and future work.

\section{RELATED WORK}

Many studies have been carried out with significant progress in the needle tracking through microscopic image [8], [9], [10]. These works achieved satisfactory results using either color-based or geometry-based features. However, due to the limitation of 2D microscopic image, these methods can not provide the enough information to reconstruct the needle including entire position and pose information.

Other 3D medical imaging technologies, including computed tomography (CT) scans, fluoroscopy, magnetic resonance (MR) or ultrasound technology are already applied in the brain, thoracic and cardiac surgeries, not only for diagnostic procedures but also as a real time surgical guide [11], [12], [13]. However, these imaging technologies can hardly achieve the ideal resolution for ophthalmic surgery application. For MRI-guided interventions with resolution in millimeters in breast and prostate biopsies, $18 \mathrm{G}$ needle, which has the diameter of $1.27 \mathrm{~mm}$, is used, while for ophthalmic surgery, $30 \mathrm{G}$ needle, which has the diameter of $0.31 \mathrm{~mm}$, requires resolution of submillimeter [14].

Some researchers used OCT for distance estimation between the surgical tool and eye tissue. Song et al. [15] developed a robotic-surgical tool with an OCT probe inside to estimate the one-dimensional distance between surgical tool and eye tissue. Yu et al. [16], and Liu et al. [17] applied the OCT probe to assist robotic ophthalmic surgery. However, they focused on integrating the OCT into the surgical system, rather than estimating the position and pose information of surgical instrument.

In this paper, based on the geometric features of beveled needle and its imaging characteristic in OCT cube, a framework is proposed and evaluated to obtain the position and pose of needle for ophthalmic surgery.

\section{METHOD}

\section{A. The framework for proposed method}

We define several parameters for the beveled needle in OCT cube, see Fig. 1(a), the center point of needle bevel $\mathbf{C}\left(c_{x}, c_{y}, c_{z}\right)$, space vector $\mathbf{n}$, and the rotation angle $\varphi$. Once we obtain the value of these three parameters, the position and pose of needle can be determined. The beveled needle has geometrical characteristics for its shape of projection on one certain plane shown in Fig. 1(b). When the needle rotation angle $\varphi$ changes from 0 to $2 \pi$, the projection of needle outline is always formed by two lines and one ellipse, where two lines are parallel and tangent to the ellipse simultaneously.

The basic idea of our proposed method is to calculate the projection of needle on the $X O Y$ plane, so that the diameter of the needle, the projection point of $\mathbf{C}$ and the rotation of the needle can be obtained. Thereafter we map these parameters

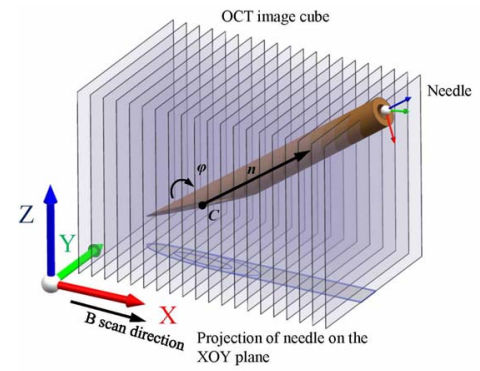

(a)

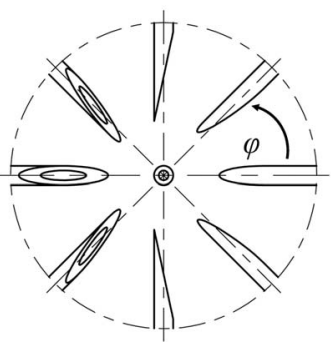

(b)
Fig. 1. (a) The position and pose of beveled needle in OCT image cube and (b) geometrical characteristics for the projection of the beveled needle.

back to 3D space to get the position and pose of the needle. The framework of our approach is shown in Fig. 2.

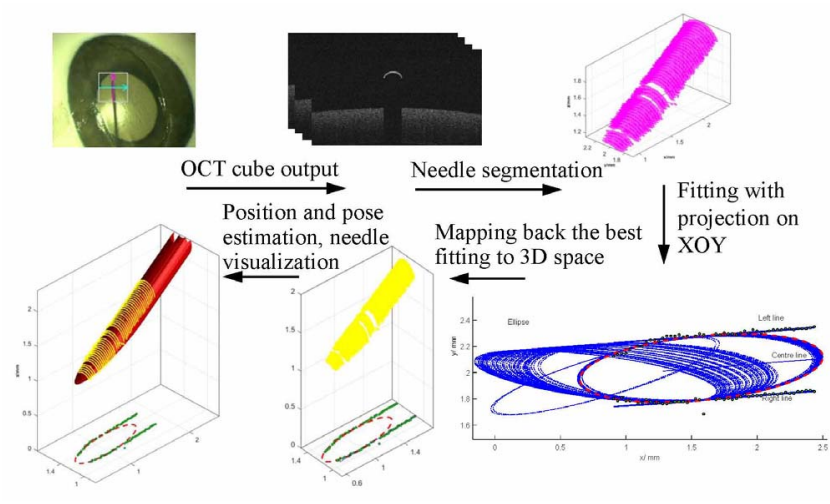

Fig. 2. The framework of the method.

\section{B. Needle segmentation and projection}

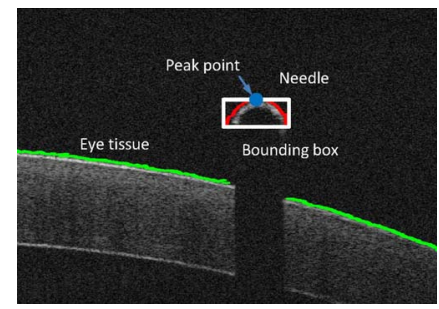

(a)

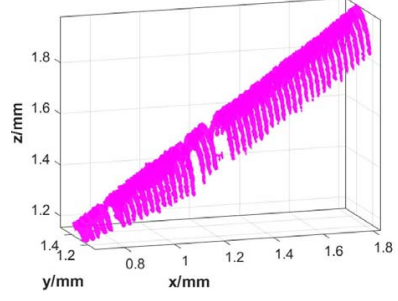

(b)
Fig. 3. (a) The needle segmentation in OCT B-scan image and (b) The 3D needle point cloud from OCT image cube.

Based on the algorithm from our previous work [18], [19], needle fragment can be robustly segmented in each B-scan image with dissolution of noise influence, see Fig. 3(a). The position of needle point can be calculated from the index of OCT B-scan and pixels position in current OCT B-scan image. The needle points can be enveloped by the bounding box $\mathbf{B}_{i}=$ $\left(B x_{i}, B y_{i}, B z_{i}, w_{i}, h_{i}\right),(i=1, \ldots, n)$, where $\left(B x_{i}, B y_{i}, B z_{i}\right)$ is the position for the top left corner of bounding box, $w_{i}$ and $h_{i}$ are the width and height of the box, and $n$ is the total number of bounding box. The peak point of needle in each B-scan image is regarded as the midpoint on the top edge of bounding box.

The projection of needle bounding box on the $X O Y$ plane is shown in Fig. 4(a). Two tangency points locate on each line, 
which can be defined as the left line and the right line based on the direction of $\mathbf{n}$. In other words, the two tangency points divide the projection point set into three point sets, ellipse point set $\mathbf{P}_{e}$, left line point set $\mathbf{P}_{l}$, and right line point set $\mathbf{P}_{r}$ with number of points $m_{e}, m_{l}$, and $m_{r}$, respectively. We define the one assignment solution as $\mathbf{P}_{i}=\left\{\mathbf{P}_{e}, \mathbf{P}_{l}, \mathbf{P}_{r}\right\}$. Width of needle $w_{i}$ will increase with $B x_{i}$ and keep constant after reaching the needle body. The break point for this relationship is one tangency point with the index of $c$ where piecewiselinear fitting method is used to calculate this index. The fitting result is shown in Fig. 4(b) with the input data of Fig. 4(a).

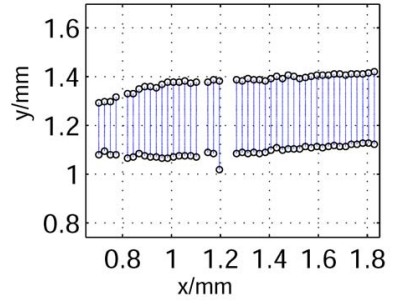

(a)

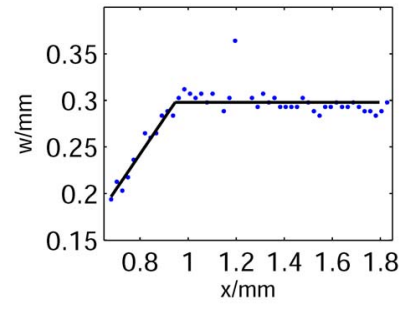

(b)
Fig. 4. (a) The projection of the needle bounding box on the $X O Y$ plane and (b) piecewise-linear fitting to find one of the tangency point.

After this procedure, only tangency point on one side line is coarsely calculated. For the other side of tangency point $c^{\prime}$, we have $c^{\prime} \in\{1, \ldots, c\}$. In order to increase the robustness of the fitting result, the single point $\mathrm{c}$ is extended to $\mathbf{c}$ by including number of $t_{0}$ symmetrical adjacent points $\mathbf{c}=\{c-$ $\left.t_{0}, \ldots, c, \ldots, c+t_{0}\right\}$. With $c$ and $c^{\prime}$ on the two sides, the points are assigned for one ellipse and two lines.

\section{Fitting with each point set}

The arbitrary ellipse on the plane XOY can be decided by five parameters. It can be regarded as transferring from a standard ellipse that its major axis and minor axis locate on the coordinates. Assume the standard ellipse is,

$$
\overline{\mathbf{x}}^{T} \mathbf{A} \overline{\mathbf{x}}=\lambda_{1} \lambda_{2}
$$

We introduce new coordinates $\mathbf{x}=\mathbf{Q} \overline{\mathbf{x}}+\mathbf{Z}$, thus rotating and shifting the ellipse. Since $\mathbf{Q}$ is an orthogonal matrix, $\mathbf{Q}^{-1}=$ $\mathbf{Q}^{T}$. Then the equation becomes,

$$
f_{e}(\mathbf{x})=(\mathbf{x}-\mathbf{Z})^{T} \mathbf{Q} \mathbf{A} \mathbf{Q}^{T}(\mathbf{x}-\mathbf{Z})-\lambda_{1} \lambda_{2}=0
$$

where $\mathbf{x}=(x, y), \mathbf{A}=\operatorname{diag}\left(\lambda_{2}, \lambda_{1}\right), \mathbf{Z}=\left(c_{x}, c_{y}\right)$, and $\mathbf{Q}=$

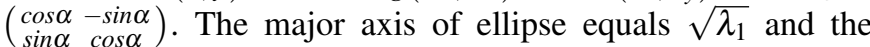
minor axis equals $\sqrt{\lambda_{2}}$.

Assume that the function for the center line of needle projection is $y=k_{0} x+b_{0}$, then the right line and left line can be presented as $f_{l}(\mathbf{x})=y-k_{0} x+b_{1}=0$ and $f_{r}(\mathbf{x})=$ $y-k_{0} x+2 b_{0}-b_{1}=0$, respectively, see Fig. 7. Before we fit the point sets with three geometric equations, following constraints need to be satisfied as:

$$
\left\{\begin{array}{l}
\text { (1) } \lambda_{1}>\lambda_{2}>0, c_{x}>0, c_{y}>0, \pi>\alpha \geq 0 \\
\text { (2) } c_{y}=k_{0} c_{x}+b_{0} \\
\text { (3) } \lambda_{1}=\left(\left(b_{1}-b_{0}\right)^{4}-\lambda_{2}(\cos \alpha+k \sin \alpha)^{2}\right)( \\
\sin \alpha-k \cos \alpha)^{-2}
\end{array}\right.
$$

It is a nonlinear least squares problem for ellipse fitting and linear least squares problem for line fitting. The subject function for algebraic distance of ellipse can be obtained by determining the parameters from equation $f_{e}(\mathbf{x})$ directly. Let $\mathbf{u}_{e}=\left(c_{x}, c_{y}, \lambda_{1}, \lambda_{2}, \alpha\right)$ be a vector of unknown parameters for ellipse and consider the nonlinear system of $m_{e}$ equations $f_{e}^{j}\left(\mathbf{u}_{e}\right)=0$, where $f_{e}^{j}\left(\mathbf{u}_{e}\right)$ is the $f_{e}\left(\mathbf{u}_{e}\right)$ with index of $j$ point in $\mathbf{P}_{e}$. With the condition $m_{e}>5$, we can minimize the sum of squares for $f_{e}^{j}\left(\mathbf{u}_{e}\right)$ with Guass-Newton method to obtain the best fitting ellipse with parameters $\tilde{\mathbf{u}}_{e}$,

$$
\tilde{\mathbf{u}}_{e}=\operatorname{argmin}_{\mathbf{u}_{e}} \sum_{j=1}^{m_{e}} f_{e}^{j}\left(\mathbf{u}_{e}\right)^{2}
$$

Assume the least square parameters for the left and right line are $\tilde{\mathbf{u}}_{l}=\left(k_{0}, b_{1}\right)$ and $\tilde{\mathbf{u}}_{r}=\left(k_{0}, b_{0}\right)$, thus the algebraic distance for point $j$ in $\mathbf{P}_{l}$ and $\mathbf{P}_{r}$ are represented as $f_{l}^{j}\left(\tilde{\mathbf{u}}_{l}\right)$ and $f_{r}^{j}\left(\tilde{\mathbf{u}}_{r}\right)$. Afterwards we can obtain the overall algebraic distance $A_{\mathbf{P}_{i}}$ for point set $\mathbf{P}_{i}$ with $\tilde{\mathbf{u}}_{e}, \tilde{\mathbf{u}}_{l}$, and $\tilde{\mathbf{u}}_{r}$,

$$
A_{\mathbf{P}_{i}}=\sum_{j=1}^{m_{e}} f_{e}^{j}\left(\tilde{\mathbf{u}}_{e}\right)^{2}+\sum_{j=1}^{m_{l}} f_{l}^{j}\left(\tilde{\mathbf{u}}_{l}\right)^{2}+\sum_{j=1}^{m_{r}} f_{r}^{j}\left(\tilde{\mathbf{u}}_{r}\right)^{2}
$$

We calculate all $\mathbf{P}_{i}$ and select the minimum overall distance for the best fitting to three parts. The fitting results using one ellipse and two lines with input data from Fig. 4(a) under $t_{0}=2$ are shown in Fig. 5. Fig. 6 presents the relationship between $A_{\mathbf{P}_{i}}$ and iteration times of Guass-Newton method, which shows that a less of $A_{\mathbf{P}_{i}}$ always contributes a less iteration times.

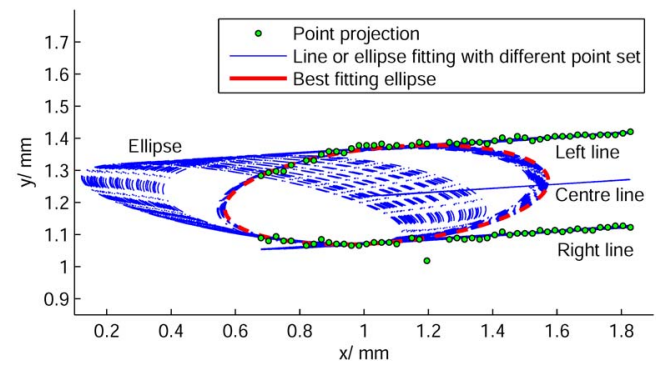

Fig. 5. The fitting result using one ellipse and two lines.

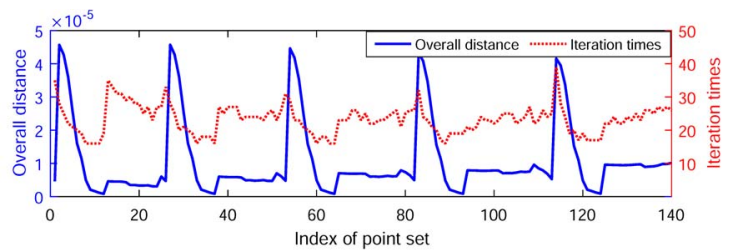

Fig. 6. The iteration times with each point set in algebraic distance and geometric distance method.

\section{The needle pose and position calculation}

In what follows, we will introduce how to map the projection with best fitting parameters back to the 3D space. The diameter $d$ and bevel angle $\beta$ of the needle can be calculated to verify the dimension of the needle. Due to the fact that the cross-sectional of needle body is a half ellipse, the center point of this ellipse can be estimated. Assume the 
equation for $l$ is $\frac{x-x_{0}}{n_{x}}=\frac{y-y_{0}}{n_{y}}=\frac{z-z_{0}}{n_{z}}$, where $\mathbf{N}=\left(x_{0}, y_{0}, z_{0}\right)$ is an arbitrary point on the $l$. We project $l$ on $X O Y$ and $X O Z$ plane. After the projection, the equation can be transformed as $y=\frac{x-x_{0}}{n_{x}} n_{y}+y_{0}=k_{0} x+b_{0}$ and $z=\frac{x-x_{0}}{n_{z}} n_{z}+z_{0}=k_{0}^{\prime} x+b_{0}^{\prime}$, where $k_{0}^{\prime}$ and $b_{0}^{\prime}$ are the parameters of the line which is fitted by the projection of center points on the $\mathrm{XOZ}$.

To simplify the equations, we let $x_{0}=0$ and $n_{x}=1, \mathbf{N}=$ $\left(0, b_{0}, b_{0}^{\prime}\right)$, then

$$
\mathbf{n}=\left(n_{x}, n_{y}, n_{z}\right)=\left(1, k_{0}, k_{0}^{\prime}\right)
$$

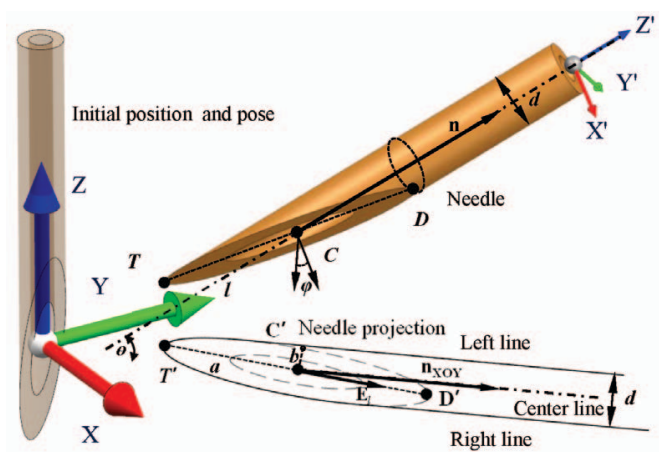

Fig. 7. The geometric parameters of the beveled needle.

The diameter of the needle $d$ is the distance between the right line and left line. The equation is listed as follows,

$$
d=\frac{2\left|b_{1}-b_{0}\right|}{\sqrt{1+k_{0}^{2}}}
$$

The center point $\mathbf{C}$ is obtained by mapping the center point of ellipse $\mathbf{C}^{\prime}$ back to the central axis line of the needle $l$. Therefore we can obtain,

$$
\mathbf{C}=\left(c_{x}, c_{y}, c_{z}\right)=\left(c_{x}, c_{x} k_{0}+y_{0}, c_{x} k_{0}^{\prime}+z_{0}\right)
$$

The rotation angle $\varphi$ can also be derived by above known parameters. $\arccos \left(\frac{2 b}{d}\right)$ can represent the angle between the minor axis of ellipse on the bevel and XOY plane. However, in the four quadrants, $\arccos \left(\frac{2 b}{d}\right)$ varies from 0 to $\frac{\pi}{2}$, shown in Fig. 1(b). In terms of quadrant of $\varphi$, we analyse it as follows. Shown in Fig. 7, assume $\mathbf{E}_{l}$ is the long axis of ellipse vector having the smaller angle with the vector $\mathbf{n}_{X O Y}$ which is the projection of $\mathbf{n}$ on the plane $X O Y$, then $\mathbf{E}_{l}$ on which side of $\mathbf{n}_{X O Y}$ can be obtained by the right hand rule. Assume $\mathbf{B}_{c}\left(b_{x}, b_{y}, b_{z}\right)$ is the central point of the needle bounding box in the section image which is the closest to point $\mathbf{C}$ in image cube, and $h_{c}$ is the height of this bounding box. If the condition $b_{z}-c_{z}>\chi h_{c}$ is met, the bevel of needle is downward and $\varphi$ locates in the second and third quadrant, where $\chi$ is a factor and here we assume $\chi=0.4$, otherwise the bevel of needle is upward and $\varphi$ locates in the first and fourth quadrant. Therefore $\varphi$ can be calculated as,

$$
\left\{\begin{array}{l}
\arccos \left(\frac{2 b}{d}\right), \text { if } \mathbf{n}_{X O Y} \times \mathbf{E}_{l} \leq 0 \& b_{z}-c_{z}>\chi h_{c} \\
\pi-\arccos \left(\frac{2 b}{d}\right), \text { if } \mathbf{n}_{X O Y} \times \mathbf{E}_{l} \leq 0 \& b_{z}-c_{z} \leq \chi h_{c} \\
\pi+\arccos \left(\frac{2 b}{d}\right), \text { if } \mathbf{n}_{X O Y} \times \mathbf{E}_{l} \geq 0 \& b_{z}-c_{z} \leq \chi h_{c} \\
2 \pi-\arccos \left(\frac{2 b}{d}\right), \text { if } \mathbf{n}_{X O Y} \times \mathbf{E}_{l} \geq 0 \& b_{z}-c_{z}>\chi h_{c}
\end{array}\right.
$$

The bevel angle of the needle $\beta$ is estimated by following,

$$
\beta=\arccos \left(\frac{d}{2 \overline{\mathbf{C D}}}\right)
$$

where point $\mathbf{D}$ is on the bevel and $\overline{\mathbf{C D}}$ is the length of major axis for the ellipse on the bevel.

$\mathbf{D}^{\prime}$ is the projection point of $\mathbf{D}$ on the plane of $X O Y$ which has already been calculated. The position of $\mathbf{D}\left(d_{x}, d_{y}, d_{z}\right)$ can be calculated by mapping back to the needle cylinder. We use geometric transformation to make the $\mathbf{C}$ to the original point and the $\mathbf{n}$ coincide with $Z$ axis. The transformation of $\mathbf{D}^{\prime}$ can be calculated as,

$$
\mathbf{D}_{T}^{\prime}=\left(d_{x}^{\prime}, d_{y}^{\prime}, d_{z}^{\prime}\right)=\mathbf{D}^{\prime} \mathbf{T}\left(-c_{x},-c_{y},-c_{z}\right) \mathbf{R}_{x}\left(-\alpha_{1}\right) \mathbf{R}_{y}\left(\alpha_{2}\right)
$$

$\mathbf{T}\left(-c_{x},-c_{y},-c_{z}\right)$ is used to translate $\mathbf{C}$ to the original point, then $\mathbf{R}_{x}\left(-\alpha_{1}\right)$ is used to rotate $\mathbf{n}$ to the $Y O Z$ plane, which $\alpha_{1}$ is the angle between $\mathbf{n}$ and $Y O Z$ plane. $\mathbf{n}$ is rotated by $\mathbf{R}_{y}\left(\alpha_{2}\right)$ to coincide with $Z$ axis. Similarly the vector $\mathbf{D}^{\prime} \mathbf{D}=(0,0,1)$ can also be calculated after rotation $\mathbf{D}_{T}^{\prime} \mathbf{D}_{T}=\left(D_{x}, D_{y}, D_{z}\right)=$ $\mathbf{D}^{\prime} \mathbf{D R}_{x}\left(-\alpha_{1}\right) \mathbf{R}_{y}\left(\alpha_{2}\right)$. Then the position of point $\mathbf{D}_{T}$ which is the transformation of $\mathbf{D}$ can be calculated by solving following equations,

$$
\left\{\begin{array}{l}
x^{2}+y^{2}=\frac{d^{2}}{4} \\
d_{x}=\frac{z-d_{z}^{\prime}}{D_{z}} d_{x}+d_{x}^{\prime} \\
d_{y}=\frac{z-d_{z}^{\prime}}{D_{z}} d_{y}+d_{y}^{\prime}
\end{array}\right.
$$

where, $d_{z}$ has two solutions, which corresponds to the two points on the needle body,

$$
\begin{array}{r}
d_{z}=\left(\sqrt{D_{z}\left(d^{2}\left(D_{x}^{2}+D_{y}^{2}\right)-4\left(D_{x} d_{y}^{\prime}-D_{y} d_{x}^{\prime}\right)\right)} \pm\right. \\
\left.\left(2 d_{z}^{\prime}\left(D_{x}^{2}+D_{y}^{2}\right)-2 D_{z}\left(D_{x} d_{x}^{\prime}+D_{y} d_{x}^{\prime}\right)\right)\right) /\left(2 D_{x}^{2}+D_{y}^{2}\right)
\end{array}
$$

where, $d_{z}$ takes the higher value when $\varphi$ varies from $\frac{\pi}{2}$ to $\frac{3 \pi}{2}$, otherwise, $d_{z}$ takes the lower value. Similarly, the needle tip point $\mathbf{T}$ can also be calculated. After all of these parameters are calculated, the needle CAD model can be modeled in stl format and transformed in 3D space for visualization.

\section{EXPERIMENTAL EVALUATION}

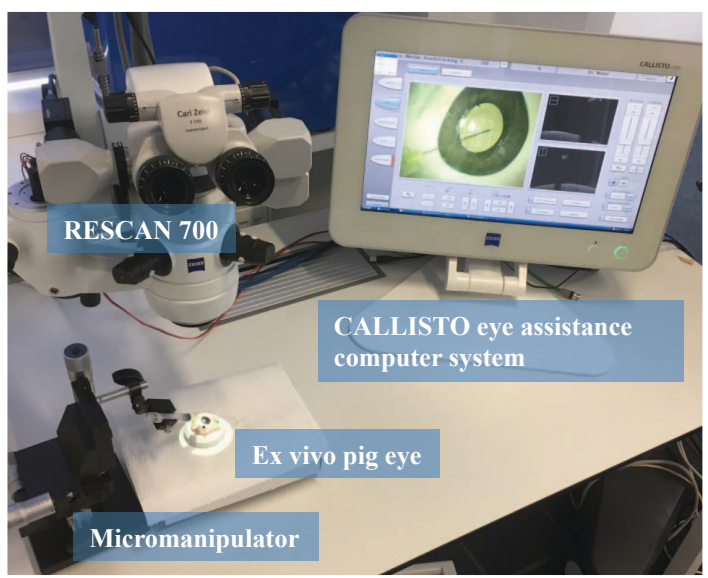

Fig. 8. Ophthalmic surgery setup with ex vivo pig eyes. The OCT and microscope are connected to the CALLISTO eye assistance computer system. The needle mounted on the manipulator is placed above the pig eye. 


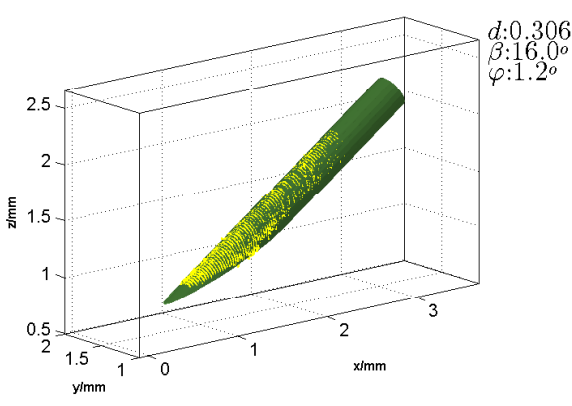

(a)

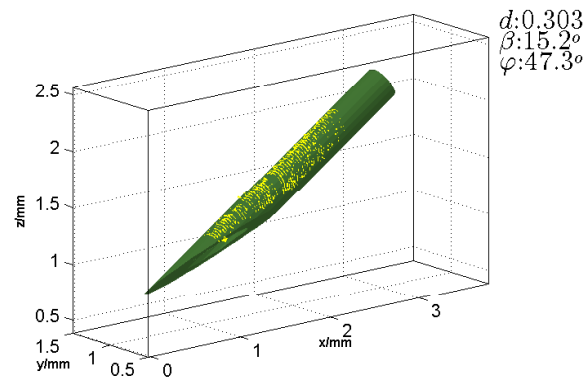

(b)

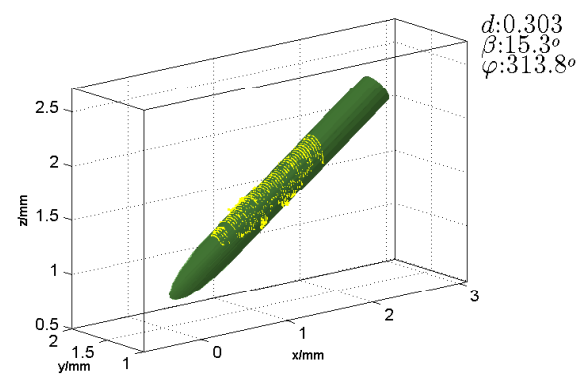

(c)

Fig. 11. The red geometry is the CAD model of beveled needle and the yellow points are the point cloud obtained by OCT. The rotation evaluation for three typical rotation angles: (a) $0^{\circ}$ rotation, (b) $45^{\circ}$ clockwise rotation, and (c) $45^{\circ}$ anticlockwise rotation.

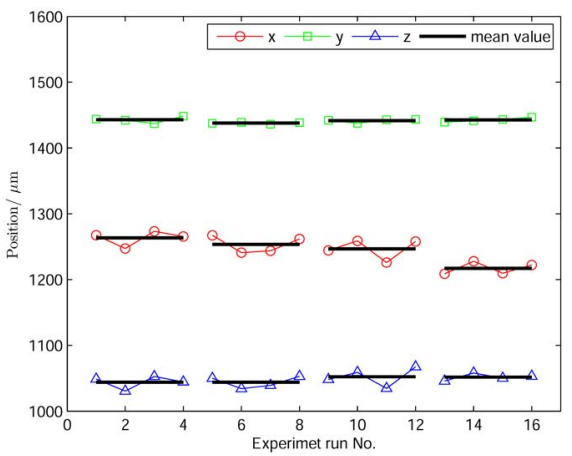

(a)

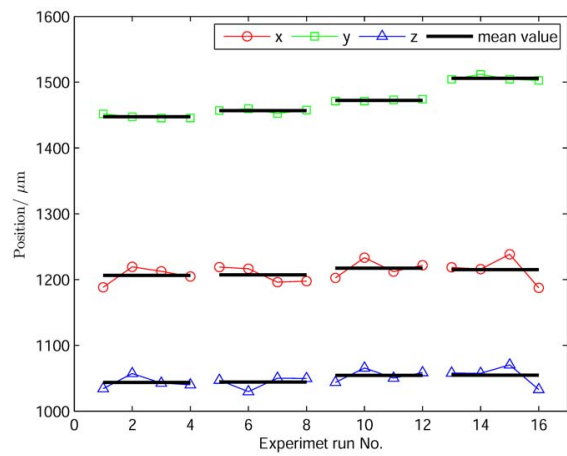

(b)

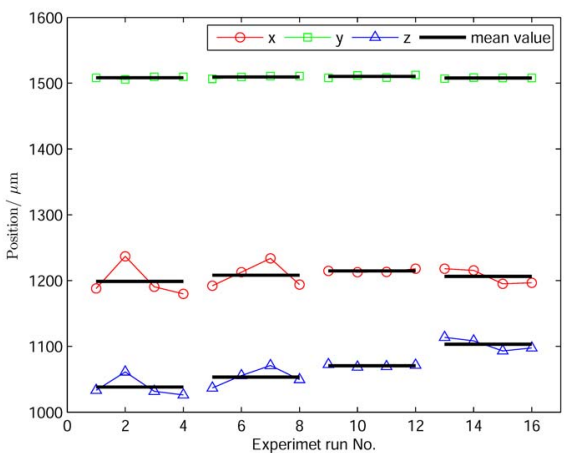

(c)

Fig. 12. The position accuracy evaluation in three directions. The position of point $\mathbf{C}$ on the needle in the OCT image cube coordinate with input $10 \mu \mathrm{m}$, $20 \mu \mathrm{m}$, and $40 \mu \mathrm{m}$ in (a) $x^{\prime}$ axis of the manipulator, (b) $y^{\prime}$ axis of the manipulator, and (c) $z^{\prime}$ axis of the manipulator.

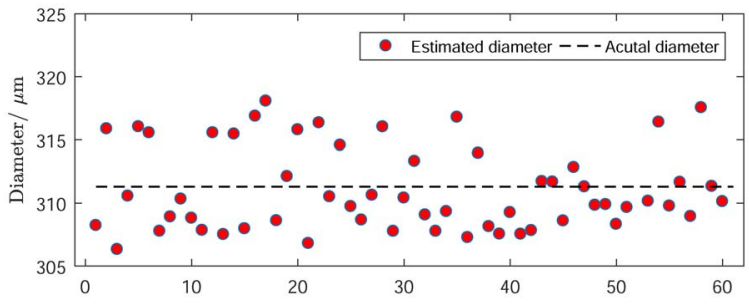

Fig. 9. The estimation of needle diameter in various of OCT cubes.

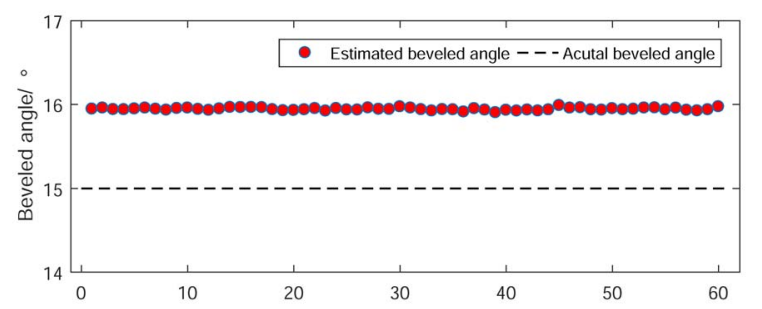

Fig. 10. The estimation of beveled angle in various of OCT cubes.

In this section, we evaluate the effectiveness and the accuracy for our proposed method of needle position and pose estimation. The ophthalmic surgery simulation setup is shown in Fig. 8. The $30 \mathrm{G}$ needle with $15^{\circ}$ bevel angle was placed close to the tissue of ex vivo pig eyes, and OCT scan area was located to include the needle tip and eye tissue. The manipulator was used to adjust the position of the needle in three directions. The CALLISTO eye assistance computer system (Carl Zeiss Meditec AG) is utilized to display the microscope image and OCT image at the same time. The image cube of OCT used in our experiments, which is the maximum resolution to be obtained from RESCAN700 currently, is 8-bit, $128 \times 512 \times 1024$ pixels represent $3 \mathrm{~mm}, 3 \mathrm{~mm}$ and $2 \mathrm{~mm}$ in $X, Y$ and $Z$ axis, respectively. The experimental evaluation contains the following parts, (1) The $30 \mathrm{G}$ needle with $15^{\circ}$ beveled is used to verify the repeatability performance of method. (2) The needle with several different rotation angle and movement in each direction are performed to show how much accuracy can be archived.

\section{A. The evaluation of repeatability performance}

The repeatability performance of proposed method with OCT imaging modality is a vital factor for the real-life application. Since the physical location of needle in OCT image cube coordinate is hard to measure, here we take the actual dimension of needle as a reference. 65 OCT image cubes with needle in several different positions are taken to estimate the diameter and beveled angle of needle, as shown in Fig. 9 and Fig. 10. The estimated diameter ranges from $306.3 \mu \mathrm{m}$ to $318.1 \mu \mathrm{m}$, while the actual diameter of the needle is $311.3 \mu \mathrm{m}$. $\beta_{A}$ keeps stable results varying from $15.9^{\circ}$ to $16.0^{\circ}$, which has a small offset with actual bevel angle. Both of the results show that the method has a good performance of repeatability. 
TABLE I

Mean distance of MOVEMENT CHANGe In OCT IMAgE CUBE AND ACTUAL MOVEMENT

\begin{tabular}{|c|c|c|c|c|c|c|}
\hline$\Delta x^{\prime}$ & $\Delta y^{\prime}$ & $\Delta z^{\prime}$ & $\Delta x$ & $\Delta y$ & $\Delta z$ & Distance \\
\hline 10 & 0 & 0 & -12.2 & -5.2 & 0.0 & 13.3 \\
\hline 20 & 0 & 0 & -8.4 & 3.8 & 8.2 & 12.4 \\
\hline 40 & 0 & 0 & -36.7 & 1.1 & -0.5 & 36.7 \\
\hline 0 & 10 & 0 & 1.1 & 9.1 & 0.6 & 9.2 \\
\hline 0 & 20 & 0 & 12.8 & 15.8 & 10.3 & 22.8 \\
\hline 0 & 40 & 0 & -2.9 & 33.4 & 0.2 & 33.5 \\
\hline 0 & 0 & 10 & 11.6 & 1.2 & 15.2 & 19.1 \\
\hline 0 & 0 & 20 & 8.1 & 0.8 & 17.2 & 19.1 \\
\hline 0 & 0 & 40 & -10.4 & -2.4 & 32.6 & 34.4 \\
\hline
\end{tabular}

\section{B. The evaluation of rotation and position accuracy}

Even though we align the coordinate system of manipulator to the imaging area as much as possible, it still can not avoid the translation and rotation between two coordinate systems. In the rotation evaluation, we rotate the needle with three typical angles and acquire the OCT image cubes. These three angles, Fig. 11(a) is the beveled plane downward, Fig. 11(b) is the needle with $45^{\circ}$ clockwise rotation, and Fig. 11(c) is the needle with $45^{\circ}$ anticlockwise rotation. Results of the needle reconstruction are presented in Fig. 11, showing that the calculated angle matches the real rotation well.

It is very hard to verify the absolute position of needle in the OCT cube coordinate system. Hence, the relative position is used to evaluate the position accuracy of needle, which can be calculated by different OCT image cubes. The manipulator is used to move the needle into three direction regards to $x^{\prime}, y^{\prime}$, and $z^{\prime}$ axis for the coordinate system of manipulator without rotating the needle. In consideration of the resolution of OCT image in each direction and weak vibration from the environment, the needle was moved in each direction with $10 \mu \mathrm{m}, 20 \mu \mathrm{m}$, and $40 \mu \mathrm{m}$. In every position, we acquire OCT cube four times. The position of the needle point $\mathbf{C}$ with movement in $x, y$, and $z$ direction is shown in Fig. 12. We obtain several interesting results from the position of needle movement. The position response calculated by OCT image cubes is quite obvious according to the actual movement input. Due to the misaligned coordinate system of the manipulator and OCT image cube, when moving the needle in one direction, there are also some changes in another direction. The fluctuation in $x$ and $z$ direction for the four repetitions is much larger than in $y$ direction, which may result from a lower resolution in $x$ direction and vibration noise from the environment. The results for mean distance of movement change in OCT image cube and actual movement are presented in Tab. I, which shows that the distance error can be controlled within $10 \mu \mathrm{m}$. This is an ideal position accuracy reported by [20] for the ophthalmic microsurgery.

\section{CONCLUSion ANd Future Work}

We proposed a novel method to reconstruct the beveled needle estimating the position and pose of needle for ophthalmic surgery using OCT image cube. The primary experiment results show that the method has a good repeatability performance and the rotation estimation matches the physical rotation angle well. The position error can be controlled within $10 \mu \mathrm{m}$ in three dimensions, which is in the range of demand accuracy level for ophthalmic microsurgery.

For future work, we plan to apply the proposed method to calibrate the coordinate system of OCT image cube with the surgical robot and navigate the needle for ophthalmic microsurgery.

\section{REFERENCES}

[1] W. H. O. (WHO), Towards universal eye health: a global action plan 2014 to 2019 report, 2013.

[2] T. Nakano, N. Sugita, T. Ueta, Y. Tamaki, and M. Mitsuishi, "A parallel robot to assist vitreoretinal surgery," IJCARS, vol. 4, no. 6, pp. 517-526, 2009.

[3] L. A. Bynoe, R. K. Hutchins, H. S. Lazarus, and M. A. Friedberg, "Retinal endovascular surgery for central retinal vein occlusion: initial experience of four surgeons," Retina, vol. 25, no. 5, pp. 625-632, 2005.

[4] N. Rieke, D. J. Tan, C. A. di San Filippo, F. Tombari, M. Alsheakhali, V. Belagiannis, A. Eslami, and N. Navab, "Real-time localization of articulated surgical instruments in retinal microsurgery," MIA, 2016.

[5] J. P. Ehlers, P. K. Kaiser, and S. K. Srivastava, "Intraoperative optical coherence tomography using the rescan 700: preliminary results from the discover study," British Journal of Ophthalmology, vol. 98, pp. 13291332., 2014.

[6] A. E. Johnson and M. Hebert, "Surface registration by matching oriented points," in 3-D Digital Imaging and Modeling, 1997. Proceedings., International Conference on Recent Advances in. IEEE, 1997, pp. 121-128.

[7] S. Gupta, P. Arbeláez, R. Girshick, and J. Malik, "Aligning 3d models to rgb-d images of cluttered scenes," in CVPR, 2015, pp. 4731-4740.

[8] Y. Li, C. Chen, X. Huang, and J. Huang, "Instrument tracking via online learning in retinal microsurgery," in MICCAI. Springer, 2014, pp. 464 471.

[9] N. Rieke, D. J. Tan, M. Alsheakhali, F. Tombari, C. A. di San Filippo, V. Belagiannis, A. Eslami, and N. Navab, "Surgical tool tracking and pose estimation in retinal microsurgery," in MICCAI. Springer, 2015, pp. 266-273.

[10] R. Sznitman, K. Ali, R. Richa, R. H. Taylor, G. D. Hager, and P. Fua, "Data-driven visual tracking in retinal microsurgery," in MICCAI. Springer, 2012, pp. 568-575.

[11] N. McDannold, G. Clement, P. Black, F. Jolesz, and K. Hynynen, "Transcranial mri-guided focused ultrasound surgery of brain tumors: Initial findings in three patients," Neurosurgery, vol. 66, no. 2, p. 323, 2010.

[12] E. R. McVeigh, M. A. Guttman, R. J. Lederman, M. Li, O. Kocaturk, T. Hunt, S. Kozlov, and K. A. Horvath, "Real-time interactive mriguided cardiac surgery: Aortic valve replacement using a direct apical approach," Magn. Reson. Med., vol. 56, no. 5, pp. 958-964, 2006.

[13] G. J. Vrooijink, M. Abayazid, and S. Misra, "Real-time threedimensional flexible needle tracking using two-dimensional ultrasound," in ICRA. IEEE, 2013, pp. 1688-1693.

[14] T. T. Lam, P. Miller, S. Howard, and T. M. Nork, "Validation of a rabbit model of choroidal neovascularization induced by a subretinal injection of fgf-lps," IVOS, vol. 55, no. 13, pp. 1204-1204, 2014.

[15] C. Song, P. L. Gehlbach, and J. U. Kang, "Active tremor cancellation by a smart handheld vitreoretinal microsurgical tool using swept source optical coherence tomography," Optics express, vol. 20, no. 21, pp. 23 414-23 421, 2012.

[16] H. Yu, J.-H. Shen, K. M. Joos, and N. Simaan, "Design, calibration and preliminary testing of a robotic telemanipulator for oct guided retinal surgery," in ICRA. IEEE, 2013, pp. 225-231.

[17] X. Liu, M. Balicki, R. H. Taylor, and J. U. Kang, "Towards automatic calibration of fourier-domain oct for robot-assisted vitreoretinal surgery," Optics express, vol. 18, no. 23, pp. 24331-24 343, 2010.

[18] H. Roodaki, K. Filippatos, A. Eslami, and N. Navab, "Introducing augmented reality to optical coherence tomography in ophthalmic microsurgery," in ISMAR. IEEE, 2015, pp. 1-6.

[19] M. Zhou, H. Roodaki, A. Eslami, G. Chen, K. Huang, M. Maier, C. P. Lohmann, A. Knoll, and M. A. Nasseri, "Needle segmentation in volumetric optical coherence tomography images for ophthalmic microsurgery," Applied Sciences, vol. 7, no. 8, p. 748, 2017.

[20] R. H. Taylor, Computer-integrated surgery: technology and clinical applications. Mit Press, 1996. 